\title{
Frequency of HLA-B5, HLA-B51 and HLA-B27 in patients with idiopathic uveitis and Behçet's disease: a case-control study
}

\author{
Saeedeh Shenavandeh ${ }^{1}$, Keramat Allah Jahanshahi ${ }^{1}$, Elham Aflaki ${ }^{1}$, Alireza Tavassoli ${ }^{2}$ \\ ${ }^{1}$ Division of Rheumatology, Department of Internal Medicine, Shiraz University of Medical Science, Iran \\ ${ }^{2}$ Department of Pathology, Fasa University of Medical Science, Shiraz, Iran
}

\begin{abstract}
Objectives: Idiopathic uveitis is the most common form of uveitis in most countries. Uveitis affects about $40-80 \%$ of patients with Behçet's disease (BD). Class I, HLA-B5, and its subclass B51 allele have the strongest association with $B D$, but its role in idiopathic uveitis is unclear. The aim of this study was to determine the frequency of HLA-B5, HLA-B51 and HLA-B27 in patients with idiopathic uveitis, BD and the control group.

Material and methods: Forty-eight patients with idiopathic uveitis, 62 patients with BD, and 49 control subjects were compared. The presence of HLA-B5, HLA-B51 and HLA-B27 was checked by reviewing the charts of patients with idiopathic uveitis and Behçet's disease referred to the rheumatologic center of Shiraz University of Medical Sciences. The control group consisted of a sex-matched normal control population, among which HLA typing was done.

Results: HLA-B5 was significantly higher in patients with idiopathic uveitis and BD compared to the control group ( $p=0.029$ and 0.0001$)$. It was significantly higher in patients with BD compared to those with idiopathic uveitis ( $p=0.001$ ). The difference of HLA-B51 between the groups was not statistically significant although it was higher in the BD group than the controls. The presence of HLA-B51 was significantly associated with ocular involvement in the BD group ( $p=0.013)$. HLA-B27 was the least common type of HLA in all groups.

Conclusions: HLA-B5 was the most common, compared to HLA-B51 and HLA-27, in patients with idiopathic uveitis and BD. There was an association between HLA-B51 positivity and ocular involvement in patients with BD. It seems that the pattern of HLA in our patients with idiopathic uveitis was different from that observed in some other studies.
\end{abstract}

Key words: Behçet’s disease, idiopathic uveitis, HLA-B5, HLA-B27, HLA-B51.

\section{Introduction}

Uveitis, the process of intraocular inflammation, may result from about 60 causes [1]. In a review study in 2011, it was shown that idiopathic uveitis was the most common cause of anterior and intermediate uveitis in Middle Eastern and European studies. Considering posterior uveitis, toxoplasmosis was the most common cause of it in these studies, while Behçet's disease (BD) and idiopathic forms were the next most common causes of posterior uveitis in the Middle East and Europe, respec- tively [2]. However, in another study in Turkey that evaluated patients with all kinds of uveitis between 1990 and 2010 retrospectively, BD was the most common etiology ahead of idiopathic uveitis [3]. It seems that idiopathic uveitis represents $23-44 \%$ of the cases with uveitis in the studies conducted in the West, Iran, Saudi Arabia, and Japan $[1,4,5]$.

Behçet's disease is a chronic relapsing inflammatory disorder of unknown etiology. Uveitis affects approximately $40-80 \%$ of patients with $\mathrm{BD}$ [6]. In a recent study in Iran on 6075 adults, eye involvement in patients with 
BD was observed in $58.1 \%$ of the participants (anterior uveitis $41.1 \%$, posterior uveitis $45 \%$, retinal vasculitis $33.6 \%$, and cataract 24.4\%) [7].

Amongst various genetic markers, class I, HLA-B5 and its subclass B51 allele have been reported to have the strongest association with $\mathrm{BD}$, but their roles in idiopathic uveitis are unclear [8-10]. The association of HLA-B51 with various manifestations of BD has been reported to be conflicting, partly due to different regiona and ethnic backgrounds in different studies.

However, a meta-analysis showed that HLA-B51 positive BD patients revealed a higher prevalence of uveitis than negative patients [6]. In some reports, HLA-B27 is the most common type of HLA in patients with idiopathic uveitis, especially in the anterior uveitis, and it is mostly prognostic because it renders the patients prone to recurrent disease [11-13]. Moreover, another study showed that the patients with BD manifesting as uveitis with a double positive test for both HLA-B27 and HLA-B51 constituted a benign subgroup of these patients and positivity of HLA-B27 may be a good prognostic factor in them [14]. This study aimed to determine the frequency of HLA-B5, HLA-B51 and HLA-B27 in our patients with idiopathic uveitis and those with BD compared with the control group.

\section{Material and methods}

In this study, 48 patients with idiopathic uveitis referred by ophthalmologists to our rheumatology clinics of Motahary and Hafez hospitals as referral centers of rheumatology in Shiraz were enrolled. Sixty-two patients with BD who fulfilled the Behçet's disease International Study Group criteria [15] and were registered in those clinics were selected by a convenience and sequential sampling method; also, 49 healthy candidates in Shiraz city, as blood donors, participated in this study.

The inclusion criteria for patients with idiopathic uveitis were pre-evaluation by an ophthalmologist specialized in retina assessment to exclude any systemic, infectious and genetic causes of uveitis, and also pre-evaluation by a rheumatologist to exclude known rheumatic diseases such as BD, spondyloarthropathies and other vasculitis causes of uveitis as well as sarcoidosis. In addition, they were required to have a follow-up chart with the diagnosis of idiopathic uveitis on the first referral and follow-up.

Patients with idiopathic uveitis and BD were examined by an ophthalmologist for evaluation of the type and location of uveitis and pattern of ocular involvement. Informed consent was obtained from all the participants included in this study.
Presence of HLA-B5, HLA-B51 and HLA-B27 was checked by reviewing the charts of patients with idiopathic uveitis and BD referring to the rheumatology center of Shiraz University of Medical Sciences. The control group was selected from the normal control population referred to Blood Donation Center of Shiraz, as a referral center. Patients and control subjects were matched for sex and the HLA typing was done for them, as well. The procedure of HLA serologic typing for HLA-B5, HLA-B51 and HLA-B27 was performed using the complement-dependent cytotoxicity (CDC) test or microlymphocytotoxicity assay. All kits and reagents were provided by Chimi-teb from Inno-train Company. Lymphocytes were separated from heparinized blood samples using Ficoll Hypaque. After washing in Hanks solution, an appropriate volume of lymphocyte suspension was poured into "Terasaki plates" and incubated with a panel of HLA-specific alloantibodies. After incubation, cells were treated with lyophilized rabbit complement (Inno-train). Lysed cells were stained with eosin and identified by an inverted microscope.

The consent of the bioethical commission was obtained for conducting the presented research.

\section{Statistical analysis}

Descriptive statistics, the $\chi^{2}$ test and the t-test were used to analyze the data in SPSS-19 software. A difference was considered significant if the $p$-value was $<0.05$.

\section{Results}

This study was conducted on 48 patients with idiopathic uveitis (group I), 62 patients with BD (group II), and 49 control subjects (group III). The mean age in group I was $39.9 \pm 11.32$ years; in the BD and control group, it was $36.69 \pm 0.5$ and $38.2 \pm 8.86$ years, respectively. There was no significant difference in the mean age between groups ( $p=0.164)$.

Group I consisted of 21 males (M) and 27 females (F), group II consisted of $28 \mathrm{M}$ and $34 \mathrm{~F}$, and group III consisted of $21 \mathrm{M}$ and $28 \mathrm{~F}$. There was no statistically significant difference among all groups in gender distribution $(p=0.970)$.

In group I, 24 patients had bilateral uveitis and 24 patients had unilateral uveitis. In group II 25 patients (40.3\%) had ocular involvement. Of these patients, 12 had anterior uveitis, 7 posterior uveitis, 4 had panuveitis, and 2 others had intermediate uveitis. Five subjects with ocular involvement also had retinal vasculitis simultaneously. Thirteen patients had bilateral uveitis and 12 patients had unilateral uveitis. Recurrent oral aphthous ulcer was seen in all 62 (100\%) BD patients, genital aphthous in 58 (93.54\%), skin manifestations in 21 (33.87\%), and 
Table I. Frequency of the pattern of uveitis in patients with Behçet's disease and idiopathic uveitis

\begin{tabular}{|lccc|}
\hline Variable & Behçet's group $(n=25)$ & Idiopathic uveitis group $(n=48)$ & \multirow{2}{*}{$p$-value* } \\
\cline { 2 - 3 } & $n(\%)$ & $n(\%)$ & 0.129 \\
\hline Anterior uveitis & $12(48)$ & $14(29.16)$ & 0.269 \\
\hline Intermediate uveitis & $2(8)$ & $1(2.08)$ & 0.093 \\
\hline Posterior uveitis & $7(28)$ & $5(10.42)$ & 0.001 \\
\hline Panuveitis & $4(16)$ & $28(58.34)$ & \\
\hline
\end{tabular}

${ }^{*} p$-values for differences in frequency of different types of uveitis in patients with Behçet's disease compared to patients with idiopathic uveitis

Table II. HLA-B5, HLA-B51 and HLA-B27 positive patients among patients with idiopathic uveitis and with Behçet's disease

\begin{tabular}{|lccccc|}
\hline Variable & \multicolumn{2}{c}{ Idiopathic uveitis } & \multicolumn{2}{c|}{ Behçet's disease $^{*}$} & \multirow{2}{*}{$p$-value $^{*}$} \\
\cline { 2 - 5 } & $N$ & $n(\%)$ & $N$ & $n(\%)$ & \\
\hline HLA-B5 positive & 48 & $13(27.08)$ & 62 & $36(58.1)$ & 0.001 \\
\hline HLA-B51 positive & 48 & $12(25)$ & 62 & $21(33.88)$ & 0.213 \\
\hline HLA-B27 positive & 46 & $2(4.35)$ & 47 & $3(6.38)$ & 0.014 \\
\hline
\end{tabular}

* $p$-values for differences in frequency of three types of HLA in patients with idiopathic uveitis compared to patients with Behçet's disease $N$ - number of patients tested for HLA-B5/B51/B27 presence

$n$-number of HLA-B5/B51/B27-positive patients

Table III. Comparison of HLA results in patients with idiopathic uveitis and in control group

\begin{tabular}{|lccccc|}
\hline Variable & \multicolumn{2}{c}{ Idiopathic uveitis } & \multicolumn{2}{c|}{ Control group } & \multirow{2}{*}{$p$-value* } \\
\cline { 2 - 5 } & $N$ & $n(\%)$ & $N$ & $n(\%)$ & \\
\hline HLA-B5 positive & 48 & $13(27.08)$ & 62 & $5(10.2)$ & 0.029 \\
\hline HLA-B51 positive & 48 & $12(25)$ & 62 & $7(14.29)$ & 0.141 \\
\hline HLA-B27 positive & 46 & $2(4.35)$ & 62 & $1(2.04)$ & 0.287 \\
\hline
\end{tabular}

* $p$-values for differences in frequency of three types of HLA in patients with idiopathic uveitis compared to control group

$N$-number of patients tested for HLA-B5/B51/B27 presence

$n$-number of HLA-B5/B51/B27-positive patients

the pathergy test was positive in 40 (64.51\%) patients with BD.

In this study, panuveitis was the most common pattern of uveitis in patients with idiopathic uveitis (58.3\%), and anterior uveitis was the most common pattern in patients with BD (48\%). There was a significant difference in the pattern of ocular involvement between the idiopathic uveitis group and BD group ( $p=0.005$ ) (Table I).

HLA-B5 was positive in $13(27.08 \%)$ cases of the idiopathic uveitis group, 36 (51.6\%) cases of the BD group, and 5 (10.2\%) cases of the control group. It was significantly higher in patients with idiopathic uveitis compared to the control group. In addition, it was significantly higher in patients with BD compared to the idiopathic uveitis and control groups (Tables II-IV). In addition, it was non-significantly higher in patients with BD with ocular involvement compared to BD patients without ocular involvement (Table V).

HLA-B51 was positive in 12 (25\%) cases in group I, $21(33.88 \%)$ cases in group II, and 7 cases (14.29\%) in group III (control). HLA-B51 was significantly higher in patients with BD compared to the control group. However, it was not significantly higher in patients with idiopathic uveitis than the control group (Tables II-IV). In addition, the prevalence of HLA-B51 was significantly higher in patients with $\mathrm{BD}$ with ocular involvement compared to BD patients without ocular involvement (Table V).

HLA-B27 was positive in 2 (4.35\%) patients with idiopathic uveitis, 3 (6.38\%) patients with BD, and 1 (2.04\%) member of the control group. It was significantly higher in patients with BD compared to the idiopathic uveitis and control groups. Although the number of HLA-B27 
Table IV. Comparison of HLA results in patients with Behçet's disease and in control group

\begin{tabular}{|c|c|c|c|c|c|}
\hline \multirow[t]{2}{*}{ Variable } & \multicolumn{2}{|c|}{ Behçet's disease } & \multicolumn{2}{|c|}{ Control group } & \multirow[t]{2}{*}{$p$-value* } \\
\hline & $N$ & $n(\%)$ & $N$ & $n(\%)$ & \\
\hline HLA-B5 positive & 62 & $36(58.1)$ & 62 & $5(10.2)$ & 0.0001 \\
\hline HLA-B51 positive & 62 & $21(33.88)$ & 62 & 7 (14.29) & 0.015 \\
\hline HLA-B27 positive & 47 & $3(6.38)$ & 62 & $1(2.04)$ & 0.001 \\
\hline
\end{tabular}

* $p$-values for differences in frequency of three types of HLA in patients with Behçet's disease compared to control group $N$ - number of patients tested for HLA-B5/B51/B27 presence

$n$-number of HLA-B5/B51/B27-positive patients

Table V. Association of HLA-B5, HLA-B51, and HLA-B27 with ocular involvement in patients with Behçet's disease

\begin{tabular}{|lccccc|}
\hline Variable & \multicolumn{2}{c}{$\begin{array}{c}\text { Behçet's disease } \\
\text { with ocular involvement }\end{array}$} & $\begin{array}{c}\text { Behçet's disease } \\
\text { without ocular involvement }\end{array}$ & \multirow{2}{*}{$p$-value* } \\
\cline { 2 - 5 } & $N$ & $n(\%)$ & $N$ & $n(\%)$ & 0.068 \\
\hline HLA-B5 positive & 25 & $18(72)$ & 37 & $18(48.6)$ & 0.013 \\
\hline HLA-B51 positive & 25 & $13(52)$ & 37 & $8(21.62)$ & 0.967 \\
\hline HLA-B27 positive & 25 & $1(5.2)$ & 37 & $2(7.1)$ & \\
\hline
\end{tabular}

* $p$-values for differences in frequency of three types of HLA in patients with Behçet's disease with ocular involvement compared with patients with Behçet's disease without ocular involvement $N$ - number of patients tested for HLA-B5/B51/B27 presence $n$-number of HLA-B5/B51/B27-positive patients

positive patients was very low (Tables II-IV), there was no difference in the prevalence of HLA-B27 between patients with BD with and without ocular involvement (Table V).

In group I, only HLA-B5 was higher than the control group and this difference was significant (Table III). In group II, HLA-B5, HLA-B51 and HLA-B27 were higher than those of the control group and this difference was significant (Table IV). There was no statistically significant relationship between the pattern of ocular involvement and HLA-B5 ( $p=0.352)$, HLA-B51 ( $p=0.933)$ or HLA-B27 $(p=0.929)$ in patients with BD.

\section{Discussion}

Based on the results of the current study, panuveitis was the most common pattern of uveitis in the idiopathic uveitis group and anterior uveitis was the most common type of uveitis in the BD group.

Anterior uveitis was the most common pattern of idiopathic and secondary uveitis in most studies around the world [2, 16-19]. In addition, in a study conducted in Iran on 475 patients, the most common form of uveitis was anterior uveitis and it was mostly idiopathic [4]. In the Al-Mezaine et al. study panuveitis was the most common pattern of uveitis in all of their patients (66.6\%) [20]. Although in our study anterior uveitis was the most common type of uveitis in all patients, when we divided the patients into BD and idiopathic uveitis groups, it was the most common pattern in the BD group and in the idiopathic group panuveitis was the most common one.

According to the results of our study, uveitis was unilateral in $50 \%$ of the subjects in the idiopathic uveitis group and in the other half it was bilateral, but in the Rahimi et al. study [4], unilateral uveitis was found in $61.4 \%$ and bilateral uveitis in $38.6 \%$ of the subjects.

In our study, in the BD group HLA-B5 was the most prevalent among studied HLA (58.1\%) That was the same for BD in the Davatchi et al. study (54\%) in Iran [7]. In some papers, there were reports of a significant association between HLA-B5 and BD compared to the control group, similar to our observations [7, 9, 21, 22]. Based on the results of the current study, there was a nearly significant relationship between HLA-B5 and eye involvement of BD. The study by Maldini et al. [23] also showed that in BD, HLA-B5 was associated with higher prevalence of eye involvement and also genital aphthosis.

In our study, we also found that HLA-B5 was higher in patients with idiopathic uveitis (27\%) than in the control group and HLA-B27 was less common among studied HLA. In a study on HLA and uveitis, it was shown that in patients with recurrent, acute, unilateral, anterior uveitis, in nearly $80 \%$ of them HLA-B27 was positive; hence, it was recommended that this HLA should be used in rou- 
tine work ups of anterior uveitis [1, 24]. However, there is no recommendation for checking HLA-B5 for evaluation of patients with idiopathic uveitis at present [1].

HLA-B51 was positive in $33.8 \%$ of the BD, $25 \%$ of the uveitis group and $14.29 \%$ of the controls in our study. This was nearly in the same range in patients with idiopathic uveitis and BD and higher than those in the control group. In the Davatchi et al. study [7], it was positive in $48.9 \%$ of the BD patients; this range was higher than in our study. The Shahaneh et al. [25] and Palmares et al. [26] studies found a significant relationship between HLA-B51 and BD, as well. In the Sakly et al. study [27], HLA-B51 was found in $16.1 \%$ of the healthy subjects and $30 \%$ of the patients with BD; this finding was compatible with the results of our study. However, in our study there was no significant association between this HLA in patients with idiopathic uveitis compared to the control group.

In addition, we found significant associations of HLA-B51 positivity and ocular involvement in our patients with BD. Moreover, this association was observed previously in another study [28].

In the current study, HLA-B27 was positive in $4.35 \%$ of the idiopathic uveitis group and $6.38 \%$ of the patients with $\mathrm{BD}$, and was the least common type of HLA in our groups. Although the presence of HLA-B27 was more frequent in patients with idiopathic uveitis than the contro group, this difference was not significant, but it was significantly higher in patients with BD. It should be mentioned that in our study, the positive HLA-B27 group was relatively small, so this relationship cannot be proven or explicitly excluded. As discussed before, in some reports, HLA-B27 was the most common type of HLA assessed in patients with uveitis and it was mostly associated with anterior uveitis [1, 8, 29].

In the present study, we evaluated the HLA typing in patients with idiopathic uveitis compared to those with $\mathrm{BD}$ and a control group. Based on the results of this study and comparison of the other reports in this field, it seems that, like previous studies, there was a significant association among HLA-B5, idiopathic uveitis and BD. HLA-B5 was the most common type of HLA in all of our uveitis patients. Due to the limited information in this field, and rather low number of patients, larger multicenter studies are suggested. Perhaps evaluation of the presence of HLAB5 should be included in the diagnosis of $\mathrm{BD}$, and as a prognostic marker of the disease, especially in patients with anterior uveitis. HLA-B5 also may be a marker of more severe, recurrent or chronic uveitis in patients with the idiopathic form of this disease. There is a need for further work-up in our high prevalence area for BD as a Silk Road country and in other countries with more immigrants from these regions.

\section{Conclusions}

HLA-B5 was the most common, compared to HLA-B51 and HLA-B27, in our patients with idiopathic uveitis and Behçet's disease.

Moreover, there was an association between HLA-B51 positivity and ocular involvement in our patients with BD. It seems that the pattern of HLA in our patients with idiopathic uveitis was different from that observed in some other studies.

\section{Acknowledgements}

The present article was extracted from a thesis written by Dr. Keramatallah Jahanshahi supported by Shiraz University of Medical Science grant number: 94-01-0111218.

The authors would like to thank Shiraz University of Medical Sciences, Shiraz, Iran, and also the Center for Development of Clinical Research of Namazee Hospital and Dr. Nasrin Shokrpour for editorial assistance, and Mr. Abolfazl Talei and Esmaeil Iranpoor, the laboratory technologists (BS), for serological HLA typing in Shiraz blood transfusion organization, special serology laboratory ward.

The authors declare no conflict of interest.

\section{References}

1. Seve P, Cacoub P, Bodaghi B, et al. Uveitis: Diagnostic work-up. A literature review and recommendations from an expert committee. Autoimmun Rev 2017; 16: 1254-1264.

2. Nashtaei EM, Soheilian M, Herbort CP, et al. Patterns of uveitis in the middle East and europe. J Ophthalmic Vis Res 2011; 6: 233-240.

3. Çakar Özdal MP, Yazici A, Tüfek M, et al. Epidemiology of uveitis in a referral hospital in Turkey. Turk J Med Sci 2014; 44: 337-342.

4. Rahimi M, Mirmansouri G. Patterns of uveitis at a tertiary referral center in southern iran. J Ohthalmic Vis Res 2014; 9: 54-59.

5. Al Dhibi HA, Al Shamsi HN, Al-Mahmood AM, et al. Patterns of Uveitis in a Tertiary Care Referral Institute in Saudi Arabia. Ocul Immunol Inflamm 2017; 25: 388-395.

6. Kang EH, Park JW, Park C, et al. Genetic and non-genetic factors affecting the visual outcome of ocular Behcet's disease. Hum Immunol 2013; 74: 1363-1367.

7. Davatchi F, Chams-Davatchi C, Shams H, et al. Adult Behcet's disease in Iran: analysis of 6075 patients. Int J Rheum Dis 2016; 19: 95-103.

8. Zamecki KJ, Jabs DA. HLA typing in uveitis: use and misuse. Am J Ophthalmol 2010; 149: 189-193.e2.

9. de Menthon M, Lavalley MP, Maldini C, et al. HLA-B51/B5 and the risk of Behcet's disease: a systematic review and metaanalysis of case-control genetic association studies. Arthritis Rheum 2009; 61: 1287-1296. 
10. Henc-Petrinovic L, Pavicic A, Dokozic M. Follow-up study of HLA-B-5-associated uveitis. Acta Med lugosl 1991; 45: 257-262.

11. Dogra M, Singh R, Agarwal A. Epidemiology of Uveitis in a Tertiary-care Referral Institute in North India. Ocul Immunol Inflamm 2017; 25(suppl 1): S46-S53.

12. Razumova IY, Godzenko AA, Vorob'eva OK, et al. Uveitis in spondyloarthritis patients and its association with HLA-B27 histocompatibility antigen: prospective study. Vestn Oftalmol 2016; 132: 4-9.

13. Monnet D, Breban M, Hudry C, et al. Ophthalmic findings and frequency of extraocular manifestations in patients with HLA-B27 uveitis: a study of 175 cases. Ophthalmology 2004; 111: 802-809.

14. Ahn JK, Park YG. Human leukocyte antigen B27 and B51 doublepositive Behçet uveitis. Arch Ophthalmol 2007; 125: 1375-1380.

15. Criteria for diagnosis of Behçet's disease. International Study Group for Behçet's Disease. Lancet (London, England) 1990; 335: 1078-1080.

16. Selmi C. Diagnosis and classification of autoimmune uveitis. Autoimmun Rev 2014; 13: 591-594.

17. Luca C, Raffaella A, Sylvia M, et al. Changes in patterns of uveitis at a tertiary referral center in Northern Italy: analysis of 990 consecutive cases. Int Ophthalmol 2018; 38: 133-142.

18. Soheilian M, Heidari K, Yazdani S, et al. Patterns of uveitis in a tertiary eye care center in Iran. Ocul Immunol Inflamm 2004; 12: 297-310.

19. Yang P, Zhang Z, Zhou H, et al. Clinical patterns and characteristics of uveitis in a tertiary center for uveitis in China. Curr Eye Res 2005; 30: 943-948.

20. Al-Mezaine HS, Kangave D, Abu El-Asrar AM. Patterns of uveitis in patients admitted to a University Hospital in Riyadh, Saudi Arabia. Ocul Immunol Inflamm 2010; 18: 424-431.

21. Karaman-Kraljevic K, Stambuk V, Stambuk N, et al. A prospective study on the etiology of uveitis. Curr Eye Res 1990; 9 Suppl: 13-16.

22. Goloeva RG, Alekberova ZS, Guseva IA, et al. Behçet's disease and associations with HLA-B5 antigen (a review of literature and the authors' findings). Ter Arkh 2010; 82: 45-49.

23. Maldini C, Lavalley MP, Cheminant M, et al. Relationships of HLA-B51 or B5 genotype with Behcet's disease clinical characteristics: systematic review and meta-analyses of observational studies. Rheumatology (Oxford) 2012; 51: 887-900.

24. Rosenbaum JT. Characterization of uveitis associated with spondyloarthritis. J Rheumatol 1989; 16: 792-796.

25. Shahneh FZ, Babaloo Z, Baradaran B, et al. Behcet's syndrome in Iranian Azari people. PJBS 2012; 15: 1045-1047.

26. Palmares J, Castro-Correia J, Coutinho MF, et al. HLA and idiopathic uveitis. Ocul Immunol Inflamm 1993; 1: 179-185.

27. Sakly N, Boumiza R, Zrour-Hassen S, et al. HLA-B27 and HLA-B51 determination in Tunisian healthy subjects and patients with suspected ankylosing spondylitis and Behçet's disease. Ann N Y Acad Sci 2009; 1173: 564-569.

28. Horie Y, Meguro A, Ohta T, et al. HLA-B51 Carriers are Susceptible to Ocular Symptoms of Behçet Disease and the Association between the Two Becomes Stronger towards the East along the Silk Road: A Literature Survey. Ocul Immunol Inflamm 2017; 25 37-40.
29. Braakenburg AM, de Valk HW, de Boer J, et al. Human leukocyte antigen-B27-associated uveitis: long-term follow-up and gender differences. Am J Ophthalmol 2008; 145: 472-479. 